\title{
COMPUTATION OF $\mathbb{Z}_{3}$-INVARIANTS OF REAL QUADRATIC FIELDS
}

\author{
HISAO TAYA
}

\begin{abstract}
Let $k$ be a real quadratic field and $p$ an odd prime number which splits in $k$. In a previous work, the author gave a sufficient condition for the Iwasawa invariant $\lambda_{p}(k)$ of the cyclotomic $\mathbb{Z}_{p}$-extension of $k$ to be zero. The purpose of this paper is to study the case $p=3$ of this result and give new examples of $k$ with $\lambda_{3}(k)=0$, by using information on the initial layer of the cyclotomic $\mathbb{Z}_{3}$-extension of $k$.
\end{abstract}

\section{INTRODUCTION}

Let $k$ be a finite totally real extension of the field of rational numbers $\mathbb{Q}$. Let $p$ be a fixed prime number and $\mathbb{Z}_{p}$ the ring of $p$-adic integers. We denote by $\lambda_{p}(k)$, $\mu_{p}(k)$ and $\nu_{p}(k)$ the Iwasawa invariants of the cyclotomic $\mathbb{Z}_{p}$-extension of $k$ for $p$ (cf. [8]). In Greenberg's paper [7], both $\lambda_{p}(k)$ and $\mu_{p}(k)$ were conjectured to be zero. However, Greenberg's conjecture is not yet proven, even for real quadratic fields, although we know by the Ferrero-Washington theorem that $\mu_{p}(k)$ is always zero when $k$ is an abelian extension of $\mathbb{Q}$ (cf. [1]).

Let $k$ be a real quadratic field and $p$ an odd prime number which splits in $k$. In a previous paper [10], we gave a sufficient condition for $\lambda_{p}(k)$ to be zero. In the present paper, we first define two invariants $n_{0}^{(r)}$ and $n_{2}^{(r)}$ for $k$ and $p$, and rewrite our previous result in terms of these invariants. Next, using this result for $p=3$, we will give some examples of $k$ with $\lambda_{3}(k)=0$, which are of a new type. For this purpose, we will compute $n_{0}^{(1)}$ and $n_{2}^{(1)}$ for $p=3$ by determining the unit group of the initial layer of the cyclotomic $\mathbb{Z}_{3}$-extension of $k$ by the method of Mäki (cf. $[3,9])$.

\section{A SUfFicient CONDITION FOR $\lambda_{p}(k)=0$}

Let $k$ be a real quadratic field with class number $h$ and fundamental unit $\varepsilon$, and $p$ an odd prime number which splits in $k$, namely, $(p)=\mathfrak{p p}^{\prime}$ in $k$ where $\mathfrak{p} \neq \mathfrak{p}^{\prime}$. Then we can choose $\alpha \in k$ such that $\mathfrak{p}^{\prime h}=(\alpha)$. Fukuda and Komatsu [5] defined two invariants $n_{1}, n_{2} \in \mathbb{N}$ for $k$ and $p$, by

$$
\mathfrak{p}^{n_{1}}\left\|\left(\alpha^{p-1}-1\right), \quad \mathfrak{p}^{n_{2}}\right\|\left(\varepsilon^{p-1}-1\right) .
$$

Here $\mathfrak{p}^{n} \| \mathfrak{a}$ means that $\mathfrak{p}^{n} \mid \mathfrak{a}$ and $\mathfrak{p}^{n+1} \nmid \mathfrak{a}$ for an ideal $\mathfrak{a}$ of $k$. Though the choice of $\alpha$ is not unique, $n_{1}$ is uniquely determined under the condition $n_{1} \leq n_{2}$.

Received by the editor October 12, 1993 and, in revised form, August 2, 1994.

1991 Mathematics Subject Classification. Primary 11R23, 11R11, 11R27, 11Y40.

Key words and phrases. Iwasawa invariants, real quadratic fields, unit groups, computation. 
For the cyclotomic $\mathbb{Z}_{p}$-extension

$$
k=k_{0} \subset k_{1} \subset k_{2} \subset \cdots \subset k_{n} \subset \cdots \subset k_{\infty},
$$

let $\mathfrak{p}_{n}^{\prime}$ be the unique prime ideal of $k_{n}$ lying above $\mathfrak{p}^{\prime}, d_{n}$ the order of $\mathfrak{p}_{n}^{\prime}$ in the ideal class group of $k_{n}$ and $E_{n}$ the unit group of $k_{n}$. For $m \geq n \geq 0$, we denote by $N_{m, n}$ the norm map from $k_{m}$ to $k_{n}$. Now we fix an integer $r \geq 0$. Then we can choose $\beta_{r} \in k_{r}$ such that $\mathfrak{p}_{r}^{\prime d_{r}}=\left(\beta_{r}\right)$. We define two other invariants $n_{0}^{(r)}, n_{2}^{(r)} \in \mathbb{N}$ for $k$ and $p$, by

$$
\mathfrak{p}^{n_{0}^{(r)}} \|\left(N_{r, 0}\left(\beta_{r}\right)^{p-1}-1\right), \quad p^{n_{2}^{(r)}}=p^{n_{2}}\left(E_{0}: N_{r, 0}\left(E_{r}\right)\right) .
$$

As in the case of $n_{1}, n_{0}^{(r)}$ is uniquely determined under the condition $n_{0}^{(r)} \leq n_{2}^{(r)}$, though the choice of $\beta_{r}$ is not unique. Put $n_{0}=n_{0}^{(0)}$, noting that $n_{2}=n_{2}^{(0)}$. It is easily seen that $n_{0} \leq n_{1} \leq n_{2}$.

Remark 1. The invariant $n_{0}^{(r)}$ is a generalization of $m_{r}$, which was defined in [10] under the assumptions that $p \nmid h$ and $n_{2} \geq 2$.

Let $A_{n}$ be the $p$-primary part of the ideal class group of $k_{n}$ and $\zeta_{p}$ a primitive $p$ th root of unity. For the $C M$-field $k^{*}=k\left(\zeta_{p}\right)$, we put $\lambda_{p}^{-}\left(k^{*}\right)=\lambda_{p}\left(k^{*}\right)-\lambda_{p}\left(\left(k^{*}\right)^{+}\right)$, where $\left(k^{*}\right)^{+}$is the maximal real subfield of $k^{*}$. Noting Theorem 1 of [5] and Lemma 3 of [10], we may rewrite Theorem 2 of [10] as follows, which is a generalization of the results of Fukuda and Komatsu in [2] and [5].

Theorem 1. Let $k$ be a real quadratic field and $p$ an odd prime number which splits in $k$. Fix an integer $r \geq 0$. Assume that

1. $A_{0}=1$,

2. $\lambda_{p}^{-}\left(k^{*}\right)=1$.

If $n_{0}^{(r)} \neq n_{2}^{(r)}$, then we have $\lambda_{p}(k)=0$.

Greenberg, Fukuda, Komatsu and Wada gave a number of examples of $k$ with $\lambda_{p}(k)=0$ in three cases where $n_{1}=1,2 \leq n_{1} \neq n_{2}$ and $n_{1}=n_{2}=2$ (cf. $\left.[2,5,6,7]\right)$. However, in the case where $n_{1}=n_{2} \geq 3$, no examples of such $k$ 's have been given until now. In the rest of this paper, we will give some examples of $k$ with $\lambda_{3}(k)=0$ in the cases where $n_{1}=n_{2}=3,4$ and 5 , using Theorem 1 for $p=3$ and $r=1$. Note that $\lambda_{3}^{-}\left(k^{*}\right)=\lambda_{3}(\mathbb{Q}(\sqrt{-3 d}))$, where $d$ is the discriminant of $k$ (cf. the proof of Theorem 10.10 in [11]). Therefore, we will compute $n_{0}^{(1)}$ and $n_{2}^{(1)}$ for $p=3$ to achieve our purpose.

\section{Computation of the invariants $n_{0}^{(1)}$ And $n_{2}^{(1)}$ FOR $p=3$}

Let $m$ be a positive square-free integer and $k=\mathbb{Q}(\sqrt{m})$. In this section, we will explain how to compute $n_{0}^{(1)}$ and $n_{2}^{(1)}$ for $p=3$. Let $k_{1}$ be the initial layer of the cyclotomic $\mathbb{Z}_{3}$-extension of $k$ and $\mathbb{Q}_{1}=\mathbb{Q}(\theta)$, where $\theta=2 \cos (2 \pi / 9)$. Then $k_{1}=\mathbb{Q}(\sqrt{m}, \theta)$. We put $\omega=(1+\sqrt{m}) / 2$ or $\sqrt{m}$ according as $m \equiv 1(\bmod 4)$ or $2,3(\bmod 4)$, and $\theta^{\prime}=2 \cos (4 \pi / 9)$. The following facts are well known:

(a) $\left\{1, \theta, \theta^{\prime}\right\}$ is an integral basis for $\mathbb{Q}_{1}$,

(b) $\{1, \omega\}$ is an integral basis for $k$.

We further assume that $m$ is prime to 3 . Since $k$ and $\mathbb{Q}_{1}$ are linearly disjoint over $\mathbb{Q}$ and their discriminants are relatively prime, we obtain the following:

(c) $\left\{1, \theta, \theta^{\prime}, \omega, \theta \omega, \theta^{\prime} \omega\right\}$ is an integral basis for $k_{1}$.

Since $k_{1}$ is a real cyclic extension of degree 6 over $\mathbb{Q}$, we can determine the unit group $E_{1}$ by Mäki's algorithm (cf. [3, 9]). We now let 


$$
E_{1}=\left\langle-1, \eta_{1}, \eta_{2}, \eta_{3}, \eta_{4}, \eta_{5}\right\rangle,
$$

where the generators $\eta_{i}$ are obtained by this algorithm and represented as linear combinations of the integral basis (c) for $k_{1}$. Then we can compute $n_{2}^{(1)}$ for $p=3$ because this algorithm enables us to determine either $N_{1,0}\left(E_{1}\right)=E_{0}$ or $E_{0}^{3}$ (cf. $\S 3$ in [9], also [3]).

Now we assume that $m \equiv 1(\bmod 3)$. In order to compute $n_{0}^{(1)}$ for $p=3$, we need to know the $\mathfrak{p}$-adic valuation of $N_{1,0}\left(\beta_{1}\right)^{2}-1$. Thus we have to find a generator $\beta_{1}$ of $\mathfrak{p}_{1}^{\prime}{ }^{d_{1}}$. Note that $d_{1}=d_{0}$ or $3 d_{0}$.

In the case where $d_{1}=3 d_{0}$, we have $\left(\beta_{0}\right)=\mathfrak{p}_{1}^{\prime 3 d_{1}}=\left(\beta_{1}\right)$. Hence, we may take $\beta_{0} \in k_{1}$ as a generator of $\mathfrak{p}_{1}^{\prime d_{1}}$ and can obtain $\beta_{0}$ explicitly by a continued fraction expansion as in solving the Pell equations.

In another case where $d_{1}=d_{0}$, we have $\left(\beta_{0}\right)=\mathfrak{p}_{1}^{\prime 3 d_{1}}=\left(\beta_{1}^{3}\right)$. Therefore we obtain

$$
\beta_{1}^{3}= \pm \eta_{1}^{r_{1}} \eta_{2}^{r_{2}} \eta_{3}^{r_{3}} \eta_{4}^{r_{4}} \eta_{5}^{r_{5}} \beta_{0} \quad \text { for some } r_{i} \in \mathbb{Z}
$$

Since $\left(\beta_{0}\right)=\left(\beta_{1}^{3}\right)$, there exists an appropriate system $\left(r_{1}, r_{2}, r_{3}, r_{4}, r_{5}\right)$, where $0 \leq$ $r_{i} \leq 2$, with the property that $\eta_{1}^{r_{1}} \eta_{2}^{r_{2}} \eta_{3}^{r_{3}} \eta_{4}^{r_{4}} \eta_{5}^{r_{5}} \beta_{0}$ is a cube in $k_{1}$. Then its unique real cubic root in $k_{1}$ is a generator of $\mathfrak{p}_{1}^{\prime d_{1}}$. To find this appropriate system, we check whether a given $\eta_{1}^{r_{1}} \eta_{2}^{r_{2}} \eta_{3}^{r_{3}} \eta_{4}^{r_{4}} \eta_{5}^{r_{5}} \beta_{0}$ is a cube in $k_{1}$, using approximate values of its unique real cubic root and its conjugates, and this is verified rigorously afterwards (see Example 1).

Our programs have been executed on a Sun SPARC-station 2 using the Clanguage and on a NEC PC-9801RA using Y. Kida's UBASIC86.

Remark 2. We do not need to know the entire unit group of $k_{1}$ for our purpose. It is sufficient to know only the unit group modulo cubic.

\section{NeW EXAmples of $k$ WITH $\lambda_{3}(k)=0$}

By executing our procedure mentioned in the previous section, we will give some examples of $k$ with $\lambda_{3}(k)=0$ in the cases where $n_{1}=n_{2}=3,4$ and 5 , respectively. First, we describe the following explicit example of such a $k$ in detail.

Example 1. Let $k=\mathbb{Q}(\sqrt{8965})$ and $p=3$. Then we can easily verify that $h=$ $2\left(A_{0}=1\right), d_{0}=2, \varepsilon=402390206+8590401 \omega, \mathfrak{p}^{2}=(890+19 \omega)$ and $\mathfrak{p}^{2}=$ $(890-19 \omega)$, so $\beta_{0}=890-19 \omega$, where $\omega$ is as in $\S 3$. It follows from the $\mathfrak{p}$-adic expansions of $\varepsilon^{2}$ and $\beta_{0}^{2}$ that $n_{0}=n_{1}=n_{2}=3$. Since $\lambda_{3}^{-}\left(k^{*}\right)=\lambda_{3}(\mathbb{Q}(\sqrt{-3 \cdot 8965}))$, it also follows from Fukuda's table [4] that $\lambda_{3}^{-}\left(k^{*}\right)=1$.

On the other hand, by the method of Mäki, we obtain a system $\left\{\eta_{1}, \eta_{2}, \eta_{3}, \eta_{4}, \eta_{5}\right\}$ of fundamental units of $k_{1}$ as follows:

$$
\begin{aligned}
& \eta_{1}=\varepsilon=402390206+8590401 \omega, \\
& \eta_{2}=\theta, \\
& \eta_{3}=\theta^{\prime}, \\
& \eta_{4}=48894-36464 \theta+19152 \theta^{\prime}+1045 \omega-772 \theta \omega+416 \theta^{\prime} \omega, \\
& \eta_{5}=49939-19568 \theta-56804 \theta^{\prime}-1045 \omega+416 \theta \omega+1188 \theta^{\prime} \omega,
\end{aligned}
$$

where $\theta$ and $\theta^{\prime}$ are as in $\S 3$. We note that $\left\{\theta, \theta^{\prime}\right\}$ is a system of fundamental units of $\mathbb{Q}_{1}$, and that $\eta_{4}$ and $\eta_{5}$ are relative units of $k_{1}$. Hence it follows that $N_{1,0}\left(E_{1}\right)=E_{0}^{3}$, so we obtain $n_{2}^{(1)}=4$. 
In this case, we have $d_{1}=d_{0}=2$ by Theorem 1 for $r=0$ of [10]. Now let us put $\gamma=\eta_{1} \eta_{2}^{2} \eta_{3} \eta_{4} \eta_{5} \beta_{0}$, i.e., choose the system $(1,2,1,1,1)$ (see Remark 3 ). Then we can get approximate values $\gamma_{i}$ of the unique real cubic roots of the six conjugates of $\gamma$ as follows:

$$
\begin{aligned}
& \gamma_{1} \doteqdot-9577.8744395484425899714390649555495943 \cdots \\
& \gamma_{2} \doteqdot-0.0578252538221422012093779803554794569 \cdots \\
& \gamma_{3} \doteqdot-11423747.694177045764647496253250783415 \cdots \\
& \gamma_{4} \doteqdot-0.0001895209088981839062474670241349699 \cdots \\
& \gamma_{5} \doteqdot-13.373368069820849234894393443243885952 \cdots \\
& \gamma_{6} \doteqdot-0.0000005612408729122976653704111340309 \cdots
\end{aligned}
$$

Taking the trace from $k_{1}$ to $\mathbb{Q}$, we have

$$
\sum_{i=1}^{6} \gamma_{i} \doteqdot-11433339.000000000000000000000000000000 \cdots
$$

which is close to an integer (if it is not, then this system is not appropriate). By solving the system of equations

$$
\left\{\begin{array}{l}
\gamma_{1}=x_{0}+x_{1} \theta+x_{2} \theta^{\prime}+\left(y_{0}+y_{1} \theta+y_{2} \theta^{\prime}\right) \omega \\
\gamma_{2}=x_{0}+x_{1} \theta^{\prime}+x_{2} \theta^{\prime \prime}+\left(y_{0}+y_{1} \theta^{\prime}+y_{2} \theta^{\prime \prime}\right) \omega^{\prime} \\
\gamma_{3}=x_{0}+x_{1} \theta^{\prime \prime}+x_{2} \theta+\left(y_{0}+y_{1} \theta^{\prime \prime}+y_{2} \theta\right) \omega \\
\gamma_{4}=x_{0}+x_{1} \theta+x_{2} \theta^{\prime}+\left(y_{0}+y_{1} \theta+y_{2} \theta^{\prime}\right) \omega^{\prime}, \\
\gamma_{5}=x_{0}+x_{1} \theta^{\prime}+x_{2} \theta^{\prime \prime}+\left(y_{0}+y_{1} \theta^{\prime}+y_{2} \theta^{\prime \prime}\right) \omega \\
\gamma_{6}=x_{0}+x_{1} \theta^{\prime \prime}+x_{2} \theta+\left(y_{0}+y_{1} \theta^{\prime \prime}+y_{2} \theta\right) \omega^{\prime},
\end{array}\right.
$$

where $\theta^{\prime \prime}=2 \cos (8 \pi / 9)$ and $\omega^{\prime}=(1-\sqrt{8965}) / 2$, we obtain the following root approximately:

$$
\left(x_{0}, x_{1}, x_{2}, y_{0}, y_{1}, y_{2}\right) \doteqdot(-1885431,1396449,-745160,-40251,29812,-15908) \text {, }
$$

which is also close to an integer (if it is not, then this system is not appropriate). We then put

$$
\beta_{1}=-1885431+1396449 \theta-745160 \theta^{\prime}-40251 \omega+29812 \theta \omega-15908 \theta^{\prime} \omega .
$$

It is easy to verify that $\gamma=\beta_{1}^{3}$, so the system $(1,2,1,1,1)$ is really appropriate. Hence $\beta_{1}$ is a generator of $\mathfrak{p}_{1}^{\prime 2}$. Taking the norm from $k_{1}$ to $k$, we have $N_{1,0}\left(\beta_{1}\right)=$ $-723897967519-15454088423 \omega$. It follows from the $\mathfrak{p}$-adic expansion of $N_{1,0}\left(\beta_{1}\right)^{2}$ that $n_{0}^{(1)}=3 \neq n_{2}^{(1)}$.

Therefore we see by Theorem 1 for $r=1$ that $\lambda_{3}(\mathbb{Q}(\sqrt{8965}))=0$.

Remark 3. In Example 1, if $\left(r_{1}, r_{2}, r_{3}, r_{4}, r_{5}\right)$ is an appropriate system, then $3^{2} \theta^{2 r_{2}} \theta^{\prime 2 r_{3}}$ is a cube in $\mathbb{Q}_{1}$. On the other hand, it is easy to see that $3 \theta^{2} \theta^{\prime}$ is a cube in $\mathbb{Q}_{1}$. Hence we obtain $r_{2}=2$ and $r_{3}=1$, so that it suffices for all practical purposes to search among only $r_{1}, r_{4}$ and $r_{5}$, where $0 \leq r_{i} \leq 2$. This is often efficient in finding an appropriate system for other examples. 
Let $m$ be a positive square-free integer such that $m \equiv 1(\bmod 3)$. We executed the above procedure for the following all real quadratic fields $k=\mathbb{Q}(\sqrt{m})$ and $p=3$ :

(i) $n_{1}=n_{2}=3$ for $1 \leq m \leq 20000$ (The number of such $k$ 's is exactly 31 ).

(ii) $n_{1}=n_{2}=4$ for $1 \leq m \leq 30000$ (The number of such $k$ 's is exactly 10 ).

(iii) $n_{1}=n_{2}=5$ for $1 \leq m \leq 50000$ (The number of such $k$ 's is exactly 2 ).

The results are summarized in Tables 1, 2 and 3. In these tables, $h, A_{0}, d_{n}$ and $\lambda_{3}^{-}\left(k^{*}\right)$ are as in $\S 2$, and $D_{n}=\left\langle C l\left(\mathfrak{p}_{n}^{\prime}\right)\right\rangle \cap A_{n}$, where $C l\left(\mathfrak{p}_{n}^{\prime}\right)$ denotes the ideal class of $\mathfrak{p}_{n}^{\prime}$. The mark $*$ indicates that the assumptions (1) and (2) of Theorem 1 are satisfied for $r=1$, but we do not know whether $\lambda_{3}(k)=0$ or not, and the mark $* *$ indicates that we cannot apply Theorem 1, so we do not know whether $\lambda_{3}(k)=0$ or not. For the 17 remaining $k$ 's, i.e., exactly 11,4 and $2 k$ 's in the cases where $n_{1}=n_{2}=3,4$ and 5 , respectively, we can verify that Greenberg's conjecture is true by Theorem 1 for $r=1$.

TABLE 1 . The case where $n_{1}=n_{2}=3$ for $p=3$

\begin{tabular}{|c||c|c|c|c|c|c|c|c|c|}
\hline$m$ & $h$ & $\left|A_{0}\right|$ & $d_{0}$ & $\left|D_{0}\right|$ & $d_{1}$ & $\left|D_{1}\right|$ & $\lambda_{3}^{-}\left(k^{*}\right)$ & $\left(n_{0}^{(1)}, n_{2}^{(1)}\right)$ & $\lambda_{3}(k)$ \\
\hline 2059 & 2 & 1 & 2 & 1 & 2 & 1 & 1 & $(4,4)$ & $*$ \\
2917 & 3 & 3 & 3 & 3 & 3 & 3 & 3 & $(4,4)$ & $* *$ \\
4081 & 4 & 1 & 4 & 1 & 4 & 1 & 1 & $(4,4)$ & $*$ \\
4279 & 6 & 3 & 6 & 3 & 18 & 9 & 2 & $(3,3)$ & $* *$ \\
5062 & 1 & 1 & 1 & 1 & 1 & 1 & 1 & $(3,4)$ & 0 \\
5611 & 10 & 1 & 5 & 1 & 15 & 3 & 3 & $(3,3)$ & $* *$ \\
7006 & 12 & 3 & 12 & 3 & 12 & 3 & 3 & $(3,4)$ & $* *$ \\
7465 & 18 & 9 & 18 & 9 & 18 & 9 & 2 & $(3,4)$ & $* *$ \\
7969 & 2 & 1 & 1 & 1 & 1 & 1 & 1 & $(3,4)$ & 0 \\
8965 & 2 & 1 & 2 & 1 & 2 & 1 & 1 & $(3,4)$ & 0 \\
9895 & 10 & 1 & 10 & 1 & 10 & 1 & 1 & $(3,4)$ & 0 \\
12007 & 3 & 3 & 3 & 3 & 3 & 3 & 2 & $(3,4)$ & $* *$ \\
12313 & 1 & 1 & 1 & 1 & 1 & 1 & 1 & $(4,4)$ & $*$ \\
12421 & 1 & 1 & 1 & 1 & 1 & 1 & 1 & $(4,4)$ & $*$ \\
12553 & 1 & 1 & 1 & 1 & 1 & 1 & 1 & $(4,4)$ & $*$ \\
13939 & 2 & 1 & 2 & 1 & 2 & 1 & 1 & $(3,4)$ & 0 \\
14113 & 13 & 1 & 13 & 1 & 39 & 3 & 2 & $(3,3)$ & $* *$ \\
15802 & 2 & 1 & 2 & 1 & 2 & 1 & 1 & $(3,4)$ & 0 \\
16081 & 2 & 1 & 1 & 1 & 1 & 1 & 1 & $(4,4)$ & $*$ \\
16519 & 1 & 1 & 1 & 1 & 1 & 1 & 1 & $(3,4)$ & 0 \\
17431 & 1 & 1 & 1 & 1 & 3 & 3 & 2 & $(3,3)$ & $* *$ \\
17443 & 1 & 1 & 1 & 1 & 3 & 3 & 3 & $(3,3)$ & $* *$ \\
17686 & 2 & 1 & 1 & 1 & 3 & 3 & 2 & $(3,3)$ & $* *$ \\
18022 & 1 & 1 & 1 & 1 & 1 & 1 & 1 & $(4,4)$ & $*$ \\
18085 & 2 & 1 & 2 & 1 & 6 & 3 & 2 & $(3,3)$ & $* *$ \\
18091 & 2 & 1 & 1 & 1 & 1 & 1 & 1 & $(3,4)$ & 0 \\
18721 & 10 & 1 & 5 & 1 & 5 & 1 & 1 & $(4,4)$ & $*$ \\
18766 & 2 & 1 & 1 & 1 & 1 & 1 & 1 & $(3,4)$ & 0 \\
18787 & 1 & 1 & 1 & 1 & 1 & 1 & 1 & $(3,4)$ & 0 \\
18826 & 6 & 3 & 6 & 3 & 6 & 3 & 2 & $(4,4)$ & $* *$ \\
19309 & 1 & 1 & 1 & 1 & 1 & 1 & 1 & $(3,4)$ & 0 \\
\hline
\end{tabular}


TABLE 2. The case where $n_{1}=n_{2}=4$ for $p=3$

\begin{tabular}{|c||c|c|c|c|c|c|c|c|c|}
\hline$m$ & $h$ & $\left|A_{0}\right|$ & $d_{0}$ & $\left|D_{0}\right|$ & $d_{1}$ & $\left|D_{1}\right|$ & $\lambda_{3}^{-}\left(k^{*}\right)$ & $\left(n_{0}^{(1)}, n_{2}^{(1)}\right)$ & $\lambda_{3}(k)$ \\
\hline 2149 & 1 & 1 & 1 & 1 & 1 & 1 & 1 & $(5,5)$ & $*$ \\
9814 & 2 & 1 & 2 & 1 & 2 & 1 & 1 & $(5,5)$ & $*$ \\
10849 & 1 & 1 & 1 & 1 & 1 & 1 & 1 & $(5,5)$ & $*$ \\
16861 & 4 & 1 & 2 & 1 & 2 & 1 & 1 & $(4,5)$ & 0 \\
17707 & 1 & 1 & 1 & 1 & 1 & 1 & 1 & $(5,5)$ & $*$ \\
24007 & 1 & 1 & 1 & 1 & 1 & 1 & 1 & $(4,5)$ & 0 \\
24985 & 2 & 1 & 2 & 1 & 6 & 3 & 3 & $(4,4)$ & $* *$ \\
25597 & 2 & 1 & 1 & 1 & 1 & 1 & 1 & $(4,5)$ & 0 \\
26245 & 16 & 1 & 4 & 1 & 4 & 1 & 1 & $(4,5)$ & 0 \\
26893 & 3 & 3 & 1 & 1 & 1 & 1 & 1 & $(4,5)$ & $* *$ \\
\hline
\end{tabular}

TABLE 3 . The case where $n_{1}=n_{2}=5$ for $p=3$

\begin{tabular}{|c||c|c|c|c|c|c|c|c|c|}
\hline$m$ & $h$ & $\left|A_{0}\right|$ & $d_{0}$ & $\left|D_{0}\right|$ & $d_{1}$ & $\left|D_{1}\right|$ & $\lambda_{3}^{-}\left(k^{*}\right)$ & $\left(n_{0}^{(1)}, n_{2}^{(1)}\right)$ & $\lambda_{3}(k)$ \\
\hline 22333 & 1 & 1 & 1 & 1 & 1 & 1 & 1 & $(5,6)$ & 0 \\
42205 & 2 & 1 & 2 & 1 & 2 & 1 & 1 & $(5,6)$ & 0 \\
\hline
\end{tabular}

\section{ACKNOWLEDGMENT}

The author wishes to express his thanks to Professor T. Fukuda for helpful suggestions during his computational work, and to Professors N. Adachi, S. Iyanaga and S. Nakajima for their kind advice and encouragement.

\section{REFERENCES}

1. B. Ferrero and L. C. Washington, The Iwasawa invariant $\mu_{p}$ vanishes for abelian number fields, Ann. of Math., 109 (1979), pp. 377-395. MR 81a:12005

2. T. Fukuda, Iwasawa $\lambda$-invariants of certain real quadratic fields, Proc. Japan Acad., 65A (1989), pp. 260-262. MR 91b:11115

3. gata Univ., Nat. Sci., 13, no.1 (1992), pp. 27-33. MR 93g:11108

4. Iwasawa $\lambda$-invariants of imaginary quadratic fields, J. of the College of Industrial Technology, Nihon Univ. 27 (1994), 35-88.

5. T. Fukuda and K. Komatsu, On $\mathbb{Z}_{p}$-extensions of real quadratic fields, J. Math. Soc. Japan, 38 (1986), pp. 95-102. MR 87d:11081

6. T. Fukuda, K. Komatsu, and H. Wada, A remark on the $\lambda$-invariants of real quadratic fields, Proc. Japan Acad., 62A (1986), pp. 318-319. MR 88a:11113

7. R. Greenberg, On the Iwasawa invariants of totally real number fields, Amer. J. Math., 98 (1976), pp. 263-284. MR 53:5529

8. K. Iwasawa, On $\mathbb{Z}_{l}$-extensions of algebraic number fields, Ann. of Math., 98 (1973), pp. 246326. MR 50:2120

9. S. Mäki, The determination of units in real cyclic sextic fields, in Lecture Notes in Math. 797. Springer-Verlag, Berlin, Heidelberg, New York (1980). MR 82a:12004

10. H. Taya, On the Iwasawa $\lambda$-invariants of real quadratic fields, Tokyo J. Math., 16 (1993), pp. 121-130. MR 94f: 11113

11. L. C. Washington, Introduction to Cyclotomic Fields, in Graduate Texts in Math. vol. 83. Springer-Verlag, New York, Heidelberg, Berlin (1982). MR 85g:11001

Department of Mathematics, School of Science and Engineering, Waseda University 3-4-1, Okubo Shinjuku-Ku, TOKYo 169, Japan

E-mail address: taya@cfi.waseda.ac.jp 